\title{
Frequency-Specific Optogenetic Deep Brain Stimulation of Subthalamic Nucleus Improves Parkinsonian Motor Behaviors
}

\author{
Chunxiu Yu, ${ }^{1,2}$ Isaac R. Cassar, ${ }^{1}$ Jaydeep Sambangi, ${ }^{1}$ and ${ }^{\circledR}$ Warren M. Grill ${ }^{1,3,4,5}$ \\ ${ }^{1}$ Department of Biomedical Engineering, Duke University, Durham, North Carolina 27708, ${ }^{2}$ Department of Biological Sciences, Michigan \\ Technological University, Houghton, Michigan 49931, ${ }^{3}$ Department of Neurobiology, ${ }^{4}$ Department of Surgery, Duke University \\ Medical Center, Durham, North Carolina 27710, and ${ }^{5}$ Department of Electrical and Computer Engineering, Duke University, Durham, \\ North Carolina 27708
}

Deep brain stimulation (DBS) of the subthalamic nucleus (STN) is an effective therapy for the motor symptoms of Parkinson's disease (PD). However, the neural elements mediating symptom relief are unclear. A previous study concluded that direct optogenetic activation of STN neurons was neither necessary nor sufficient for relief of parkinsonian symptoms. However, the kinetics of the channelrhodopsin-2 (ChR2) used for cell-specific activation are too slow to follow the high rates required for effective DBS, and thus the contribution of activation of STN neurons to the therapeutic effects of DBS remains unclear. We quantified the behavioral and neuronal effects of optogenetic STN DBS in female rats following unilateral 6-hydroxydopamine (6-OHDA) lesion using an ultrafast opsin (Chronos). Optogenetic STN DBS at 130 pulses per second (pps) reduced pathologic circling and ameliorated deficits in forelimb stepping similarly to electrical DBS, while optogenetic STN DBS with ChR2 did not produce behavioral effects. As with electrical DBS, optogenetic STN DBS exhibited a strong dependence on stimulation rate; high rates produced symptom relief while low rates were ineffective. High-rate optogenetic DBS generated both increases and decreases in firing rates of single neurons in STN, globus pallidus externa (GPe), and substantia nigra pars reticular (SNr), and disrupted $\beta$ band oscillatory activity in STN and SNr. High-rate optogenetic STN DBS can indeed ameliorate parkinsonian motor symptoms through reduction of abnormal oscillatory activity in the STN-associated neural circuit, and these results highlight that the kinetic properties of opsins have a strong influence on the effects of optogenetic stimulation.

Key words: Deep Brain Stimulation; Frequency-specific; Optogenetics; Parkinson's Disease; Subthalamic Nucleus

\section{Significance Statement}

Whether STN local cells contribute to the therapeutic effects of subthalamic nucleus (STN) deep brain stimulation (DBS) in Parkinson's disease (PD) remains unclear. We re-examined the role of STN local cells in mediating the symptom-relieving effects of STN DBS using cell type-specific optogenetic stimulation with a much faster opsin, Chronos. Direct optogenetic stimulation of STN neurons was effective in treating the symptoms of parkinsonism in the 6-hydroxydopamine (6-OHDA) lesion rat. These results highlight that the kinetic properties of opsins can have a strong influence on the effects of optogenetic activation/inhibition and must be considered when employing optogenetic to study high-rate neural stimulation.

\footnotetext{
Received Dec. 30, 2019; revised Apr. 1, 2020; accepted Apr. 7, 2020

Author contributions: C.Y. and W.M.G. designed research; C.Y., I.R.C., and J.S. performed research; C.Y. and I.R.C. analyzed data; C.Y. and W.M.G. wrote the paper.

This work was partially supported by the National Institute of Neurological Disorders and Stroke under Award Numbers National Institutes of Health R01 NS091236 and R37 NS040894 (to W.M.G.) and R03 NS108097 (to C.Y.).

The authors declare no competing financial interests.

Correspondence should be addressed to Warren M. Grill at warren.grill@duke.edu.

https://doi.org/10.1523/JNEUROSCI.3071-19.2020

Copyright $\odot 2020$ the authors
}

\section{Introduction}

Deep brain stimulation (DBS) is a surgical therapy for the motor symptoms of advanced Parkinson's disease (PD). The subthalamic nucleus (STN) is a common DBS target, and STN DBS produces remarkable improvements in resting tremor, rigidity, bradykinesia, and akinesia (Krack et al., 1998; Deep-Brain Stimulation for Parkinson's Disease Study Group et al., 2001). Despite well-established clinical benefits, how STN DBS exerts its therapeutic effects remains unclear, and this limits treatment optimization, as well as potential applications of DBS in other neurologic and psychiatric disorders. 
Brain tissue is anatomically heterogeneous and fiber-dense, and DBS could modulate the activity of multiple neuronal elements in target sites and adjacent areas (Alexander et al., 1990; Albaugh and Shih, 2014). Given this complexity, understanding the neural elements that contribute to therapeutically effective DBS will enable improvements to this treatment. Application of cell-type-specific optogenetic DBS led to the conclusion that the therapeutic effects of STN DBS do not result from stimulation of neurons in the STN, but rather result from antidromic activation of the (hyperdirect) cortical input to STN (Gradinaru et al., 2009). However, the finding that optogenetic activation of STN neurons did not ameliorate parkinsonian symptoms, and the subsequent conclusion that antidromic activation of the hyperdirect pathway is the therapeutic target of STN DBS, are unclear because the slow response kinetics of channelrhodopsin-2 (ChR2) are incapable of maintaining high-rate firing, a critical parameter for effective STN DBS (Boyden et al., 2005; Lin et al., 2009; Yizhar et al., 2011; Jun and Cardin, 2020). During highrate stimulation, ChR2 expressing neurons respond with irregular patterns of activity with firing rates well below the rates of optical stimulation (Boyden et al., 2005; Lin et al., 2009; Hight et al., 2015). Importantly, random patterns of electrical STN DBS are not effective at treating parkinsonian symptoms in either the 6-hydroxydopamine (6-OHDA) lesioned rat (McConnell et al., 2016) or in humans with PD (Dorval et al., 2010). Thus, it remains unclear which neural elements and circuits are essential for the therapeutic benefits of STN DBS.

Here, we used optogenetic STN DBS with an ultrafast opsin, Chronos, to re-assess the effects of selective STN DBS on behavior and neural activity. Chronos exhibits much faster kinetics than ChR2 (Klapoetke et al., 2014; Jun and Cardin, 2020) and is able to follow high-rate [ $>100$ pulses per second (pps)] stimulation with much greater fidelity (Hight et al., 2015). Chronos was packaged into an adeno-associated virus serotype 5 (AAV5) with a calcium/calmodulin-dependent protein kinase II (CaMKII) promoter to generate local cell-specific expression in STN in the 6-OHDA lesioned rat model of PD. We quantified the behavioral effects of optogenetic DBS of the STN on parkinsonian motor symptoms using circling behavior and forelimb adjusting steps. We conducted single unit recordings in STN, substantia nigra pars reticular ( $\mathrm{SNr}$ ), and globus pallidus externa (GPe) during different rates of optogenetic STN stimulation and quantified the effects of STN DBS on neural activity. Cell-specific high-rate STN optogenetic DBS alleviated pathologic circling and improved abnormal stepping, behavioral effects that were not achieved with similarly targeted optogenetic STN DBS with ChR2, and disrupted abnormal oscillatory activity in the STN and downstream neural circuits.

\section{Materials and Methods}

All animal care and experimental procedures were approved by the Institutional Animal Care and Use Committee (IACUC) at Duke University.

\section{Animals and surgery}

Nineteen female Sprague Dawley rats (250-300 g) were used for behavioral studies and electrophysiological recordings. Five rats were injected with Chronos and implanted with optical fibers in the STN; three rats were injected with ChR2 and implanted with fibers; five rats were implanted with metal microwire electrodes in the STN for electrical stimulation. Six rats were injected with Chronos and used for acute optogenetic stimulation and recording of single units. All rats received unilateral infusion of 6-OHDA into the medial forebrain bundle (MFB) to produce hemi-parkinsonism. Surgery was performed under general anesthesia with sevoflurane (induction at $7 \%$ in $2 \mathrm{l} / \mathrm{min} \mathrm{O}_{2}$; maintained at $3.5 \%$ with $\left.1.5 \mathrm{l} / \mathrm{min} \mathrm{O}_{2}\right)$. Dexamethasone $(5.0 \mathrm{mg} / \mathrm{kg}$ ) was injected subcutaneously to reduce potential for brain swelling during surgery. The body temperature was maintained at $\sim 37^{\circ} \mathrm{C}$ with a water heating blanket. Craniotomies were performed over the unilateral STN and MFB according to a stereotactic atlas of the rat brain (STN: AP $-3.6 \mathrm{~mm}$ from bregma, ML $2.6 \mathrm{~mm}$; MFB: AP $-2.0 \mathrm{~mm}$, ML $2.0 \mathrm{~mm}$ ) as previously described (McConnell et al., 2012).

\section{Viral injection and unilateral dopamine depletion}

For optogenetic experiments, the viral vectors rAAV5-CaMKII-ChronosGFP $\left(1.8 \times 10^{13} \mathrm{vg} / \mathrm{ml}\right)$ and rAAV5-CaMKII-hCHR2 (H134R)-EYFP $\left(4.6 \times 10^{12} \mathrm{vg} / \mathrm{ml}\right)$ were stereotyped and packaged by the University of North Carolina at Chapel Hill Vector Core. The virus $(0.6 \mu \mathrm{l})$ was injected into the STN (DV $-7.0 \mathrm{~mm}$ ) at a rate of $0.1 \mu \mathrm{l} / \mathrm{min}$ through a $1-\mu \mathrm{l} 32$ Gauge Hamilton syringe, waiting $5 \mathrm{~min}$ after injecting every $0.2 \mu \mathrm{l}$, and the needle was left in the brain for another $10 \mathrm{~min}$ after the full injection. Metal microelectrode recordings were used during the surgery to localize the STN before the injection. Rats were then lesioned to cause unilateral degeneration of dopaminergic neurons in the substantia nigra pars compacta $(\mathrm{SNc})$ and produce hemi-parkinsonism. The 6-OHDA $(6 \mu \mathrm{l}, 2.5 \mathrm{mg} /$ $\mathrm{ml}$ in $0.2 \%$ ascorbic acid dissolved with saline, Sigma-Aldrich) was prepared immediately before use and infused into MFB (DV $-7.5 \mathrm{~mm}$ ) through $10-\mu \mathrm{l}$ Hamilton syringe at a rate of $1 \mu \mathrm{l} / \mathrm{min}$, waiting $5 \mathrm{~min}$ after every $2 \mu \mathrm{l}$, and the needle was left in the brain for another $10 \mathrm{~min}$ after the full injection. Thirty minutes before lesioning, the rats were administered $50 \mathrm{mg} / \mathrm{kg}$ pargyline (intraperitoneally, Sigma-Aldrich) and $5 \mathrm{mg} / \mathrm{kg}$ desipramine (intraperitoneally, Sigma-Aldrich) to inhibit monoamine oxidase and protect noradrenergic neurons.

\section{Fiber implantation}

For rats with optogenetic stimulation, an optical fiber $(200-\mu \mathrm{m}$ core diameter, 1.25-mm OD ceramic zirconia ferrule, Precision Fiber Products) was lowered into the STN (DV -6.8 $\mathrm{mm}$ from bregma) and cemented in place with dental acrylic. Rats were then single housed for at least five weeks to allow time for viral expression and recovery from lesion and surgery before the behavioral paradigms began. For electrophysiological recordings with optical stimulation, the surgical openings were sutured after 6-OHDA and virus injection. The rats were allowed to recover for at least five weeks before the acute recordings.

\section{Electrode implantation}

Rats for electrical STN DBS were implanted with stimulating electrodes in STN, recording electrodes in SNr and a cannula in MFB for 6-OHDA injection. A stimulating electrode array $(2 \times 2$, platinum-iridium, $75-\mu \mathrm{m}$ diameter, $0.3-\mathrm{mm}$ interelectrode spacing, and $10-\mathrm{k} \Omega$ impedance; Microprobes) was implanted in the STN. A $4 \times 4$ recording multichannel electrode array (platinum-iridium, $75-\mu \mathrm{m}$ diameter, $0.25-\mathrm{mm}$ interelectrode spacing, $\sim 500-\mathrm{k} \Omega$ impedance; Microprobes) was implanted in the $\mathrm{SNr}$ (from bregma in $\mathrm{mm}, \mathrm{AP}-5.8, \mathrm{ML} 2.3, \mathrm{DV}-7.2$ ). A 23-Gauge stainless steel cannula was placed in the MFB (DV $-7.5 \mathrm{~mm}$ ). The implanted arrays and cannula were secured using dental acrylic attached to stainless steel bone screws anchored to the skull. After five weeks of recovery, rats were lesioned with 6-OHDA under 3.5\% sevoflurane anesthesia. The rats were pretreated with $50 \mathrm{mg} / \mathrm{kg}$ pargyline and $5 \mathrm{mg} / \mathrm{kg}$ desipramine injection (intraperitoneal) $30 \mathrm{~min}$ before the lesion. Then 6 -OHDA $(10 \mu \mathrm{l}, 2.5 \mathrm{mg} / \mathrm{ml})$ was infused through the cannula at a rate of $2 \mu \mathrm{l} / \mathrm{min}$ using a $10-\mu \mathrm{l}$ Hamilton syringe. Rats were left for recovery for at least one week before any additional measurements.

\section{Behavioral tests}

\section{Stimulation parameters}

For optogenetic experiments, after at least five weeks for viral expression and nigral neuron degeneration, rats were connected to a 473-nm DPSS laser (Shanghai Laser) via fiber optic cables and placed inside a testing chamber. Stimulation was pulsed at a fixed rate $(5,20,75,100$, and 130 pps) with a pulse width of $1 \mathrm{~ms}$ for Chronos-injected rats and $5 \mathrm{~ms}$ for ChR2-injected rats. We used 5-ms pulses for ChR2 stimulation to be 

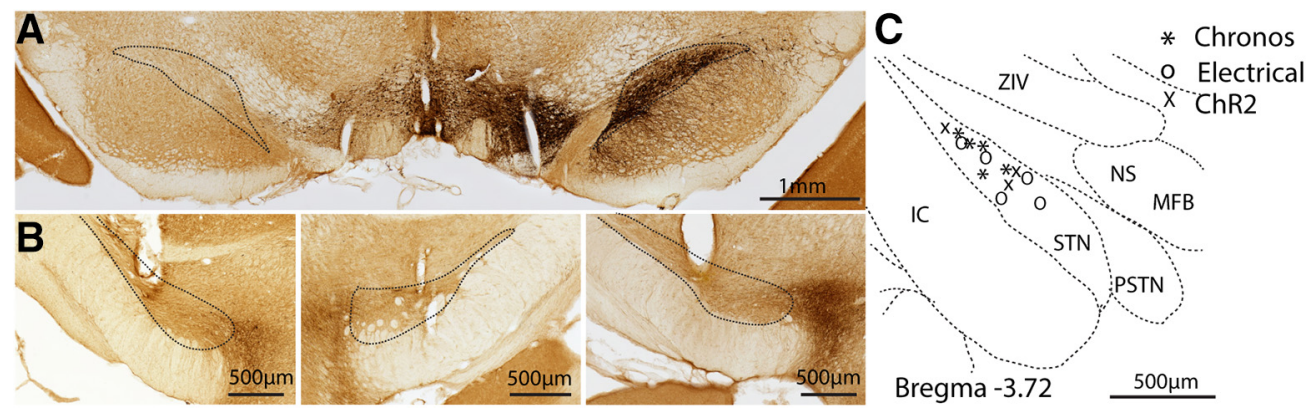

Figure 1. Verification of 6-OHDA lesion and fiber/electrode implantation in STN. $A$, Coronal section immunostained for TH verifies unilateral 6-0HDA lesion producing $>90 \%$ loss of dopaminergic neurons in the substantia nigra compacta (SNc). B, TH-stained representative coronal sections show the locations in STN of optical fiber (left, Chronos; right, ChR2) and electrode (middle) locations. C, Fiber and electrode locations in STN across all implanted rats used for circling and forelimb stepping analysis (Chronos, $n=5$; electrical, $n=5$; ChR2, $n=3$ ) on a coronal section. The diagram is based on a rat brain atlas (Paxinos and Watson, 2005). In rats with electrical stimulation, one electrode of the bipolar pair used for stimulation was within STN.
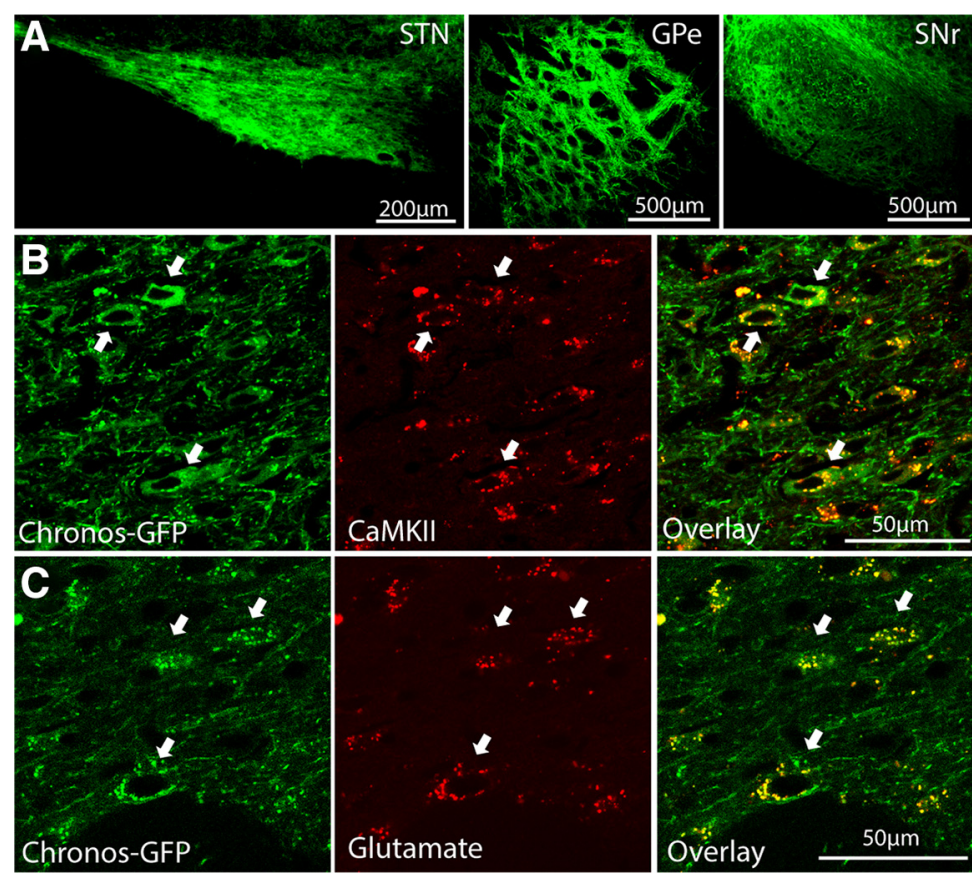

Figure 2. Expression of Chronos-GFP in STN, GPe, and SNr. A, Immunofluorescence-stained sections show neurons expressing Chronos-GFP following viral injection in STN (left), and densely labeled terminals in GPe (middle) and SNr (right). B, C, Fluorescence images show STN neurons expressing Chronos-GFP and labeled for the excitatory neuron-specific CaMKII (B) and glutamate (C). Arrows indicate Chronos-GPF positive neurons that were also immunoreactive for CaMKII or glutamate.

consistent with and thereby enable direct comparison with previous studies of optogenetic STN DBS with ChR2 (Gradinaru et al., 2009). Since Chronos has much faster off-kinetics and is able to follow reliably high rate, we used a shorter pulse width of $1 \mathrm{~ms}$. We confirmed that optogenetic STN DBS in Chronos-injected animals $(n=3)$ using 5 -ms pulses produced effects on circling and linear speed that were comparable to those with $1-\mathrm{ms}$ pulses. The fiber output was $10 \mathrm{~mW}$ measured with a PM20A photodetector (Thorlabs). For electrical DBS, stimulation with charge balanced biphasic pulses (pulse width of $90 \mu \mathrm{s} /$ phase) was conducted using an isolated voltage to current converter (A-M System) controlled by MATLAB software. The appropriate amplitude of stimulation for each rat was determined before the start of experiments based on sustained motor responses including increased contralateral turning, decreased ipsilateral turning, increased activity, and increased rearing, and a lack of motor side effects including involuntary muscle contractions of the limbs and neck during 130-pps stimulation.
$500 \mu \mathrm{m}$

\section{Circling test}

To induce robust and protracted circling, unilaterally lesioned rats received a single injection of methamphetamine $(1.875 \mathrm{mg} / \mathrm{kg}$ in $0.9 \%$ saline $) 30 \mathrm{~min}$ before placement in a cylinder (So et al., 2012). The cylinder was placed in a dark sound attenuating chamber equipped with an infrared camera to record rat behavior. A rotating optical (Doric) or electrical commutator (PlasticsOne) was used to prevent cable twisting. Behavioral effects of DBS were examined using fixed 130 pps and randomized blocks of 5 stimulation rates: 5, 20, 75,100 , and 130 pps with presentation order randomized within each block. For fixed 130-pps optical stimulation, pulses were delivered in trains of 6 trials (each trial was composed of $10 \mathrm{~s}$ on and $20 \mathrm{~s}$ off) for a total of $3 \mathrm{~min}$. Each behavioral recording session started with $3 \mathrm{~min}$ control period, followed by a 3-min stimulation period and ended with 3 min light off. The entire session was 9 min long. For rate-randomized stimulation, pulse trains were delivered for $10 \mathrm{~s}$ at each rate followed by a 20-s off interval. Each session started with at least 2-min control period, followed by the stimulation period, and ended with 2-min light off. For each rat, six sessions were recorded for both $130 \mathrm{pps}$ and the randomized rates, and the repeated sessions were performed at least $2 \mathrm{~d}$ apart. For rats with electrical DBS, four blocks of five different rates of DBS were delivered, with 60-s stimulation epochs spaced $120 \mathrm{~s}$ apart. Circling behavior was video recorded and tracked using behavioral analysis software (Clever Systems). The position of the nose and the base of tail over time were computed. Angular velocity and distance traveled per minute (linear speed) were calculated offline from the tracking data using MATLAB. Normalized angular velocity or linear speed for each trial was calculated by dividing the angular velocity or linear speed during the stimulation-on period by the average angular velocities or linear speeds during the prestimulation-off and poststimulation-off periods immediately before and after the stimulation-on period. Normalized angular velocities or linear speed for each stimulation rate were then calculated by averaging across all randomized blocks.

\section{Adjusting steps test}

Hemi-parkinsonian rats exhibit specific deficits in contralateral limb use, and deficits in forelimb adjusting steps is a validated measure of parkinsonian akinesia in rats (Schallert et al., 1978; Glajch et al., 2012). Each rat was held with their hind limbs elevated and moved backward at a steady rate traversing a 1-m glass corridor (Runway, CleverSys) over $\sim 3-4 \mathrm{~s}$. The movement was videotaped and analyzed offline by counting the number of steps made with the contralateral and ipsilateral forelimbs. Continuous optical or electrical DBS at 20- or 130-pps DBS was delivered in addition to no stimulation control trials. Two to three trials were 
recorded in each session, and this was repeated across four to six sessions. The behavioral effects of DBS were quantified by the ratio of the number of steps with the contralateral forelimb to the number of steps with the ipsilateral forelimb.

\section{Electrophysiological recording}

After at least five weeks of recovery following viral injection in STN, electrophysiological recordings in STN, GPe, and SNr were conducted in ipsilaterally lesioned rats under urethane anesthesia $(1.2 \mathrm{~g} / \mathrm{kg}$, subcutaneous). Following a midline incision of the scalp, soft tissue was retracted, and a craniotomy was drilled over STN, GPe, and SNr. The dura was removed at each craniotomy location, and the brain was covered with normal saline. A bone screw was implanted at the back of the head and served as electrical ground. Optical stimulation and unit recording in STN were conducted using a custom made optrode with an optical fiber $(200 \mu \mathrm{m})$ attached to single tungsten microelectrode $(0.5 \mathrm{M} \Omega, 75-\mu \mathrm{m}$ diameter) with the tip of the fiber $0.3-0.4 \mathrm{~mm}$ above the electrode tip and angled so the emitted light encompassed the electrode tip (Sparta et al., 2011). Sixteen channel electrode arrays were used for single unit recordings from GPe or SNr during optical stimulation in the STN. Each electrode/array was slowly advanced using a micromanipulator. Separate recording depths for each penetration were spaced 300$400 \mu \mathrm{m}$ apart. Neuronal activity was recorded using a multichannel acquisition processor system (Plexon Inc). The data were sampled at $40 \mathrm{ksamples} / \mathrm{s}$. Stimulation at $130 \mathrm{pps}$ or five randomized rates (5, $20,75,100$, and $130 \mathrm{pps}$ ) occurred in a train of 10 trials (each trial consisted of 10-s stimulation on and 20 -s stimulation off). Each session began with baseline recording for at least $60 \mathrm{~s}$ in which no stimulation was presented and ended with baseline recording of at least $60 \mathrm{~s}$.

\section{Electrophysiological data analysis}

Electrophysiological data were analyzed by a combination of custom-written MATLAB scripts. Single units were extract and sorted with the Plexon Offline Sorter using principal component analysis (Plexon Inc). Spikes were detected by applying a bandpass filter $(150 \mathrm{~Hz}$ to $9 \mathrm{kHz}$ ) to raw traces with an amplitude threshold of $-4^{*} \mathrm{SD}$ and $800-\mu \mathrm{s}$ duration snippets of spike waveforms were extracted. Only single-unit activity with a clear separation from noise was used for the analysis, and units with refractory period shorter than $1 \mathrm{~ms}$ were removed. Neural activity within a window of $-10-20 \mathrm{~s}$ aligned to stimulus onset was averaged in 200-ms bins and averaged across trials to construct peristimulus time histograms (PSTHs). The PSTHs were then $Z$-scored by subtracting the mean baseline firing rate (measured from a baseline window from -10 to $0 \mathrm{~s}$ before trial initiation) and dividing by the standard deviation. To classify "excited" versus "inhibited" units, neural activity during 10-s stimulation on was compared with a baseline window from $10 \mathrm{~s}$ before the stimulus onset (two-tailed $t$ test, $p<0.05$ ). Peak latency was computed as time elapsed between the stimulus onset and the time point within $100 \mathrm{~ms}$ when the response reached its first peak. Spectral analysis of the spike times was used to quantify temporal structure in the data. Multitaper analysis was used to construct spectral estimators (Chronux version 2.12; Mitra and Pesaran, 1999) on 10-s windows using 5 Slepian data tapers. Spectra are presented on a logarithmic scale. Spectral power in the $\beta$ band $(12-30 \mathrm{~Hz})$, low $\beta$ band $(12-20 \mathrm{~Hz})$, and high $\beta$ band $(20-30 \mathrm{~Hz})$ during the stimulation on period were calculated and compared with the average of the spectral power during the prestimulation off and poststimulation off periods immediately before and after the stimulation on period (two-tailed $t$ test, $p<0.05$ ). Power spectrograms were constructed within a window of $-10-20 \mathrm{~s}$ aligned to stimulus onset using a 1-s sliding window and 0.1-s steps.

\section{Statistical analysis}

Statistical significance between conditions was determined using a oneway or two-way repeated measures ANOVA. The normality of data were judged by frequency distributions (histogram) and boxplots (Ghasemi and Zahediasl, 2012). Post hoc paired comparisons were used if the corresponding main effect or interaction was significant at $p<0.05$. To control for multiple comparisons, pairwise post hoc tests were performed with the Tukey's HSD test with a $p<0.05$ significance cutoff. For single comparisons, two-sample Mann-Whitney test or paired $t$ test was performed. All statistical tests were performed using MATLAB. All results are presented as mean \pm SE.

\section{Histology}

Following completion of experiments, rats were deeply anesthetized with urethane $(1.8 \mathrm{~g} / \mathrm{kg}$, i.p.) and perfused transcardially with $0.1 \mathrm{M} \mathrm{PBS}$ followed by $4 \%$ paraformaldehyde in $0.1 \mathrm{M}$ PBS. Brains were postfixed in $4 \%$ paraformaldehyde overnight at $4^{\circ} \mathrm{C}$ and then transferred to $30 \%$ sucrose $\left(4^{\circ} \mathrm{C}\right)$. The brains were cut into 40 - or $50-\mu \mathrm{m}$ sections in the coronal plane (except one rat with electrical DBS was horizontally sectioned) using a cryostat (CM3050S, Leica Microsystems) and processed for staining. Tyrosine hydroxylase (TH) immunohistochemistry was used to determine the extent of degeneration of dopaminergic 

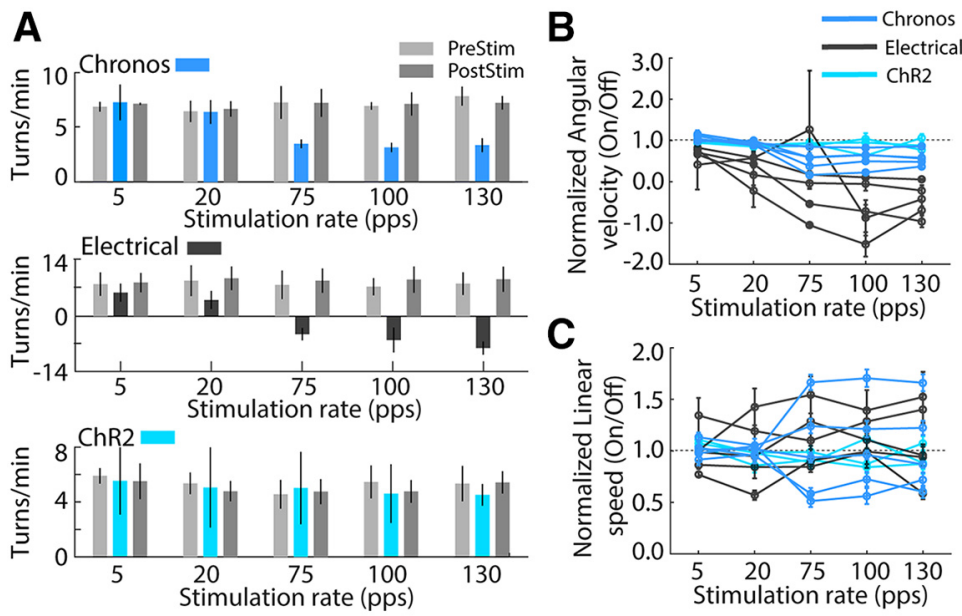

Figure 4. Behavioral effects of different rates of STN DBS on methamphetamine-induced circling in hemi-parkinsonian rats. $\boldsymbol{A}$, Effects of DBS rate on circling in representative stimulation sessions. Top, Behavioral effect of optogenetic STN DBS in Chronos-injected rat was dependent on stimulation rate. High-rate optogenetic STN DBS (75, 100, and 130 pps) suppressed ipsilateral turning while low rate (5 and 20 pps) had no effect. Middle, High-rate electrical STN DBS reduced ipsilateral turning while low rate electrical stimulation had no effect. Bottom, Optogenetic DBS in ChR2-injected rat had no behavioral effect at high or low rates. $\boldsymbol{B}$, Effects of DBS rate on circling across all Chronos $(n=5)$, electrical $(n=5)$, and ChR2 $(n=3)$ stimulated rats. Ipsilateral circling was suppressed in Chronos injected and electrically stimulated rats at high rates of stimulation (75, 100, 130 pps; all $p<0.001$, two-way ANOVA and post hoc comparison). Circling were not affected in ChR2-injected rats (all $p>0.05$ ). C, Effects of DBS rate on linear speed of movement across all Chronos $(n=5)$, electrical $(n=5)$, and ChR2 $(n=3)$ stimulated rats. There were no changes of linear speed during three type of stimulations (two-way ANOVA, all $p>0.05$ ). Data are mean $\pm \mathrm{SE}$.
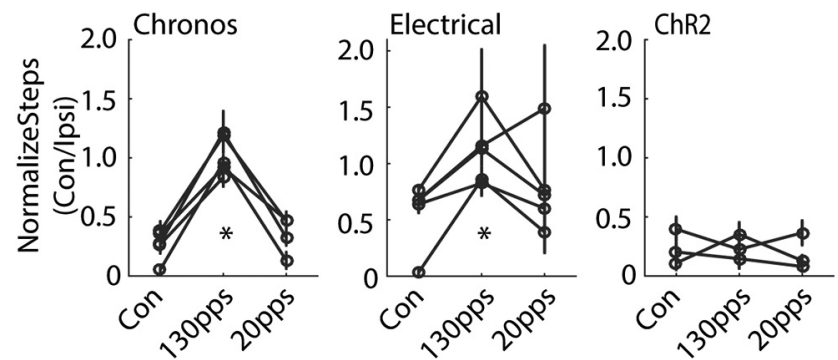

Figure 5. Behavioral effects of STN DBS on forelimb adjusting steps in hemi-parkinsonian rats. Left, High-rate optogenetic STN DBS at 130 pps in Chronos-injected rats increased the ratio of contralateral-to-ipsilateral limb use, indicating an increase of impaired forelimb use. Low-rate optogenetic STN DBS at 20 pps in Chronos-injected rats had no effect $(p<0.001$, one-way repeated measures ANOVA, $n=5$ ). Middle, High-rate STN electrical DBS increased the ratio of contralateral-to-ipsilateral limb use, but low rate electrical DBS did not $(p<0.001, n=5)$. Right, 0ptical STN DBS in ChR2-injected rats did not generate behavioral effects $(p=0.527, n=3) ;{ }^{*} p<0.001$. Data are mean \pm SE.

neurons in the SNc and electrode/fiber locations. Briefly, brain sections were first rinsed and blocked for $1 \mathrm{~h}$ in $10 \%$ goat serum, and then incubated in anti-TH antibody (AB152; 1:1000, Vector Laboratories) overnight at $4^{\circ} \mathrm{C}$ in solution with $10 \%$ goat serum and $0.25 \%$ Triton $\mathrm{X}-100$ with PBS. The sections were incubated with biotinylated goat anti-rabbit secondary antibody (BA-1000, 1:250, Vector Laboratories) with 10\% goat serum and $0.25 \%$ Triton X-100 in PBS for $1 \mathrm{~h}$ at room temperature. After rinsing, the sections were incubated in a VECTASTAIN Elite ABC kit (Vector Laboratories) solution for $1 \mathrm{~h}$ and then visualized using DAB solution. To visualize GFP positive cells, the neighboring sections were directly mounted with DAPI-FluoroMount-G (SouthernBiotech) after rinsing. To determine the cell-specific expression of Chronos-GFP, brain sections were immunostained for CaMKII or glutamate. Slices were incubated with either primary anti-CaMKII antibody (1:300, Millipore, catalog \#05-532) or primary rabbit anti-glutamate antibody (1:500, Millipore, AB133) with $10 \%$ goat serum and $0.25 \%$ Triton X-100 overnight at $4^{\circ} \mathrm{C}$. Secondary antibodies (Alexa Fluor 594 goat anti-mouse IgGI) were used to visualize CaMKII or glutamate (1:500, Life
Technologies). All sections were mounted with DAPI-FluoroMount-G (SouthernBiotech). Images were acquired with Nikon Eclipse TE2000-U, Zeiss AxioPlan 2 or Zeiss 780 inverted confocal microscope. The locations of electrodes, fibers, and injections were compared with a rat brain atlas (Paxinos and Watson, 2005).

\section{Results}

\section{Histologic verification}

We unilaterally injected 6-OHDA into MFB and produced unilateral loss of nigral dopaminergic cells indicated by dramatic reductions in $\mathrm{TH}$ immunoreactivity in the SNc of the lesioned hemisphere (Fig. 1A) in all rats used for behavioral and electrophysiological studies $(n=19)$. To assess the behavioral effects of optogenetic STN DBS, we expressed Chronos $(n=5)$ or ChR2 $(n=3)$ and implanted optical fibers in the STN. To compare the behavioral effects with traditional electrical DBS, we also implanted stimulating electrodes in the STN in five rats. The locations of implanted optical fibers $(n=8)$ and stimulating electrodes $(n=5$, at least one electrode of the bipolar pair used for stimulation) were confirmed to reside within the STN (Fig. $1 B, C$ ). We targeted Chronos-GFP driven by the CaMKII promotor to neurons in STN. The virus injection labeled neurons in the STN and their projection terminals in the GPe and SNr (Fig. 2A). We did not observe Chronos-GFP labeled neurons in the motor cortex (M1) that projects to STN, excluding retrograde transmission to M1. Chronos-GFP was co-expressed with CaMKII (Fig. 2B) and glutamate (Fig. 2C) demonstrating successful and specific targeting of excitatory neurons in the STN.

\section{Optogenetic STN DBS improved pathologic circling}

We quantified the effects of optogenetic and electrical DBS on methamphetamine-induced circling behavior. Optogenetic STN DBS at 130 pps in Chronos-injected animals reduced pathologic circling behavior as indicated by decreases in angular velocity compared with the pre-light- and post-light-stimulation periods (Fig. $3 A, B$, left; $F_{(2,545)}=67.1, p<0.001$, one-way repeated measures ANOVA). This was consistent with the results of electrical DBS, which was highly effective in reducing circling (Fig. $3 B$, middle; $F_{(2,59)}=66.4, p<0.001$, one-way ANOVA). However, optogenetic STN DBS at 130 pps in ChR2-injected animals did not influence pathologic circling (Fig. $3 B$, right; $F_{(2,305)}=1.43$, $p=0.240$ ) consistent with previous results (Gradinaru et al., 2009). The amelioration of pathologic circling during electrical DBS was significantly greater than during optogenetic DBS using Chronos (Fig. 3C; $p<0.001$, Mann-Whitney test). Further, neither electrical stimulation nor optogenetic stimulation in Chronos-injected or ChR2-injected rats produced changes in linear speed (Fig. 3D,E; Chronos: $F_{(2,545)}=0.07, p=0.935$; electrical: $\left.F_{(2,59)}=0.04, p=0.960 ; \operatorname{ChR} 2: F_{(2,305)}=1.06, p=0.346\right)$, and there were no significant differences between the three conditions ( $p>0.27$, Mann-Whitney test). Importantly, these data indicated that the reductions in angular velocity during DBS did not result from reductions in overall movement or freezing during stimulation. 
The behavioral effects of electrical STN DBS are strongly dependent on stimulation pulse rate (McConnell et al., 2012), and we applied five different rates of optogenetic STN DBS. Chronos optical DBS and electrical DBS decreased ipsilateral circling with higher stimulation rates reflected as a decrease in angular velocity compared with the off conditions [Fig. 4A,B; two-way ANOVA with significant main effects of stimulation rate $\left(F_{(4,1265)}=40.91, p<0.001\right)$ and stimulation type $\left(F_{(2,1265)}=154.48\right.$, $p<0.001)$ and a significant interaction between these factors $\left(F_{(8,1265)}=11.91\right.$, $p<0.001)$ ]. Post hoc paired comparisons revealed that optical STN DBS in Chronos-injected rats at high rates (75, $100,130$ pps; all $p<0.001)$ relieved ipsilateral turning, while low rate (5 and 20 pps) DBS was not effective. This was consistent with the effects of electrical DBS (75, 100, 130 pps; all $p<0.01)$, but not optogenetic DBS in ChR2 expressing animals (all $p>0.05$; Fig. 4B). Meanwhile, the stimulation rate had no significant main effect on linear speed (Fig. 4C; twoway ANOVA, $\left.F_{(4,1265)}=0.36, p=0.839\right)$. There was no difference between the three stimulation types (stimulation type, $\left.F_{(2,1265)}=2.98, p=0.051\right)$, and there was no significant interaction between stimulation rate and stimulation type $\left(F_{(8,1265)}=\right.$ $0.97, p=0.457)$.

\section{Optogenetic STN DBS improved forelimb stepping}

Optogenetic DBS at 130 pps in Chronosinjected rats corrected the bias for using the unimpaired forepaw, as indicated by the increases in the ratio of contralateral to ipsilateral steps, whereas optogenetic DBS at 20 pps did not improve impaired forelimb use (Fig. 5, left; $F_{(2,251)}=$ 115.5, $p<0.001$, one-way ANOVA). Similarly, electrical DBS at 130 pps increased use of the contralateral limb and corrected the stepping bias, while 20 pps electrical DBS had no effect (Fig. 5, middle; $\left.F_{(2,178)}=11.1, p<0.001\right)$. In contrast, optogenetic DBS in ChR2 expressing animals did not have any effect on the use of the impaired forepaw at either 130 or 20 pps (Fig. 5, right; $F_{(2,124)}=$ $0.64, p=0.527)$.

\section{Changes in neural activity in STN, GPe, and SNr during 130- pps optogenetic STN DBS}

Single unit neural activity was recorded from STN ( $n=34$ units) in six rats, from GPe $(n=38)$ in three rats, and from $\mathrm{SNr}(n=61)$ in three rats, and recording electrode locations were confirmed by postmortem histology (Figs. 6A, 7A, $8 A$ ).

\section{Neuron firing rates}

Optogenetic DBS at 130 pps increased or decreased neural activity in the STN (Fig. 6). Approximately 53\% of STN single units exhibited an increase in firing rate during light stimulation (excited, $n=18 / 34$ ). These neurons rapidly increased their firing rate at stimulus onset, and firing then stabilized at a higher rate for the duration of the stimulation. Meanwhile, $32 \%$ of STN neurons exhibited a decrease in firing rate (inhibited, $n=11 / 34$ ). Inhibited neurons decreased their firing rates, and spiking stabilized at lower rate for the duration of the stimulation (Fig. 6C,D). The remaining $5 \%$ of neurons $(n=5)$ did not exhibit significant changes in firing rate during light delivery. Similarly, neurons in GPe and SNr exhibited both increases and decreases in firing rate during 130-pps optogenetic STN DBS. The majority of neurons in GPe exhibited increased firing (53\%, $n=20 / 38)$, and only four neurons (11\%) exhibited decreased firing in response to light stimulation (Fig. 7). Conversely, a majority of neurons (60\%, $n=37 / 61)$ recorded in the SNr decreased their firing rates during STN stimulation, and only $20 \%$ of SNr neurons $(n=12 /$ $61)$ increased their firing rates in response to light stimulation (Fig. 8).

We quantified response latencies of units in STN, GPe, and $\mathrm{SNr}$ measured from stimulus onset to the response peak for excited units and to the response trough for inhibited neurons (Fig. 9A-C). Excited units in the STN responded rapidly to light stimulation with latencies of $12.5 \mathrm{~ms}$ (median; Fig. 9A), while inhibited units decreased their firing rates at longer latencies of $20 \mathrm{~ms}$ (median; Fig. 9A; $p=0.036$, Mann-Whitney test). This difference in latencies suggested that inhibitory responses in the STN may result from recruitment of inhibitory neurons 
A

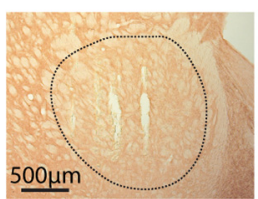

B
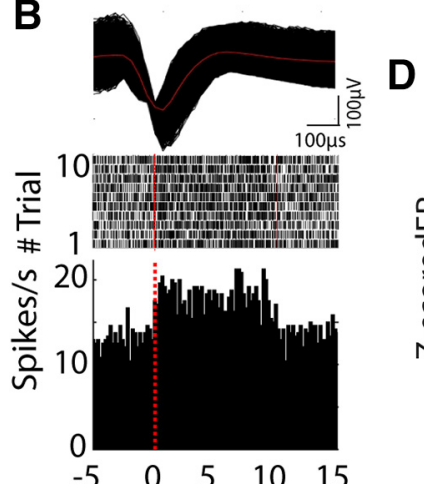

Time from stimulus on (s)
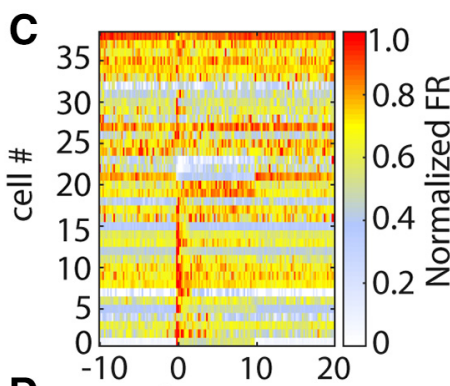

D Time from stim onset (s)

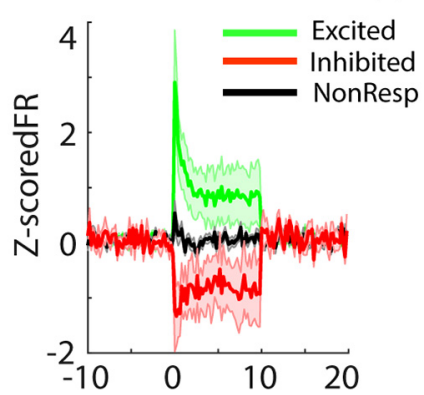

(s) Time from stim onset (s)

Figure 7. Changes in neural activity in the GPe during 130-pps optogenetic STN DBS. $\boldsymbol{A}$, TH-stained representative coronal section shows the electrode tracks within the GPe. $\boldsymbol{B}$, top, Action potential waveform of a representative GPe neuron. Middle, Rastergram of the representative neuron responding to STN optical stimulation in a Chronos-injected rat. Each row in raster represents a single trial. Bottom, Poststimulus histogram of the representative neuron. C, Heatmap of neural firing rate sorted by responses to light stimulus in STN for excited, inhibited, and non-responsive neurons; $x$-axis indicates time from stimulus onset, and each row represents the firing rate normalized by the maximum firing rate of each neuron. $\boldsymbol{D}, \mathbf{Z}$-score normalized population firing rate of excited, inhibited, and non-responsive GPe neurons.

downstream of light-activated excitatory neurons. The latencies of excited GPe neurons exhibited a bimodal distribution with a median latency of $37.5 \mathrm{~ms}$, which was significantly longer than STN excited neurons $(p<0.001)$, while the four inhibited GPe neurons had latencies of $10 \mathrm{~ms}$ (median). Both excited and inhibited neurons in the SNr exhibited longer latencies (excited, median $=25 \mathrm{~ms}$, $p=0.009$; inhibited, median $=20 \mathrm{~ms}, p=0.015)$ than STN excited neurons. The longer latencies of inhibited neurons in the SNr suggested that the suppression of neural responses in the $\mathrm{SNr}$ may result from network mechanisms involving local inhibitory recruitment and external inhibition from another input, such as GPe.

To understand further the suppression of neural activity in the STN, SNr and GPe, we conducted a correlation analysis to examine the influence of baseline firing rate on response during optogenetic DBS (Fig. 9D-F). There was a significant negative correlation between the reduction of firing rate and baseline firing rate in inhibited STN neurons $(r=-0.90, p=0.0002$, Pearson's linear correlation coefficient), but no significant correlation was found in excited STN neurons $(r=0.02, p=0.935)$. Similarly, the reductions in firing rate of inhibited neurons in $\mathrm{SNr}$ and GPe were also inversely correlated with their baseline firing rates $(\mathrm{SNr}, r=-0.60, p=0.0001$; GPe, $r=-0.99$, $p=0.008$ ). The increase in firing rate of excited neurons in $\mathrm{SNr}$ displayed significant dependence on their baseline firing rates $(r=0.61, p=0.036)$, but this was not observed for excited GPe neurons $(r=-0.20, p=0.401)$.

\section{Oscillatory activity}

Changes in neural oscillatory activity in basal ganglia circuits are associated with the parkinsonian state and altered by electrical

A
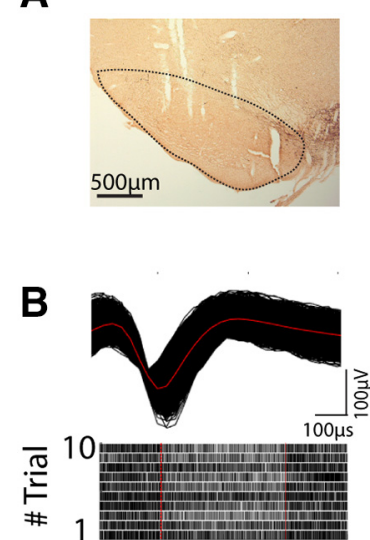

D
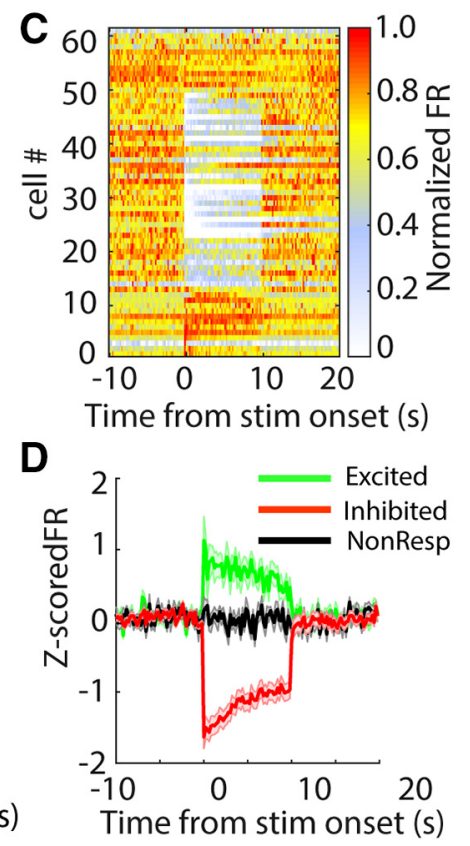

Figure 8. Changes in neural activity in the SNr during 130-pps optogenetic STN DBS. $\boldsymbol{A}$, TH-stained representative coronal section shows the electrode tracks in the SNr. $\boldsymbol{B}$, top, Action potential waveform of a representative SNr neuron in a Chronos-injected rat. Middle, Rastergram of the representative neuron responding to STN optical stimulation in a Chronosinjected rat. Each row in raster represents a single trial. Bottom, Poststimulus histogram of the representative neuron. $\boldsymbol{C}$, Heatmap of neural firing rate sorted by responses to light stimulus in STN for excited, inhibited, and non-responsive neurons; $x$-axis indicates time from stimulus onset and each row represents the firing rate normalized by the maximum firing rate of each neuron. $\boldsymbol{D}, \mathrm{Z}$-score normalized population firing rate of excited, inhibited, and non-responsive SNr neurons.

DBS. We conducted spectral analysis of single unit firing times to quantify the effects of optogenetic DBS on oscillatory activity. Before stimulation, the power spectra of STN neurons $(26 / 34$, $76 \%)$ showed a pronounced peak in the $\beta$ band $(12-30 \mathrm{~Hz})$. This oscillatory activity was entirely eliminated during optical stimulation at $130 \mathrm{pps}$ but returned to prestimulation levels after stimulation was turned off (Fig. 10A,D). 22/26 (85\%) STN neurons exhibited decreased $\beta$ band oscillatory activity during 130pps optogenetic DBS $\left(t_{(25)}=2.10, p=0.044\right.$, paired $t$ test $)$. In GPe, 25/38 recorded neurons (63\%) exhibited increased oscillatory activity in the $\beta$ band in the parkinsonian state. In contrast to STN, GPe $\beta$ oscillatory power was either unchanged or shifted to a slightly higher frequency during STN optogenetic DBS (Fig. $10 B, E ; t_{(24)}=-0.35, p=0.728$ ), and only six GPe neurons $(6 / 25,24 \%)$ showed deceased $\beta$ band activity during stimulation. The majority of $\mathrm{SNr}$ neurons $(52 / 61,85 \%)$ displayed pronounced $\beta$ band oscillatory activity before STN DBS, and this oscillatory activity was clearly decreased during optogenetic STN DBS and recovered after cessation of DBS (Fig. 10C,F); 38/ 52 (73\%) SNr neurons exhibited a reduced oscillatory peak in the $\beta$ band during 130 pps optical DBS $\left(t_{(51)}=6.59, p<0.001\right.$, paired $t$ test).

$\beta$ Oscillatory activity can be further subdivided into low-frequency $(12-20 \mathrm{~Hz})$ and high-frequency $(20-30 \mathrm{~Hz})$ components, and differential changes in the low and high $\beta$ bands may have differential effects on parkinsonian symptoms. There was reduction of high $\beta$ power in STN neurons during optogenetic STN DBS at 130 pps $\left(t_{(25)}=2.19, p=0.039\right)$, but no changes were detected in low $\beta$ band power $\left(t_{(25)}=1.09, p=0.289\right)$. Both low and high $\beta$ activity in $\mathrm{SNr}$ were significantly suppressed during 

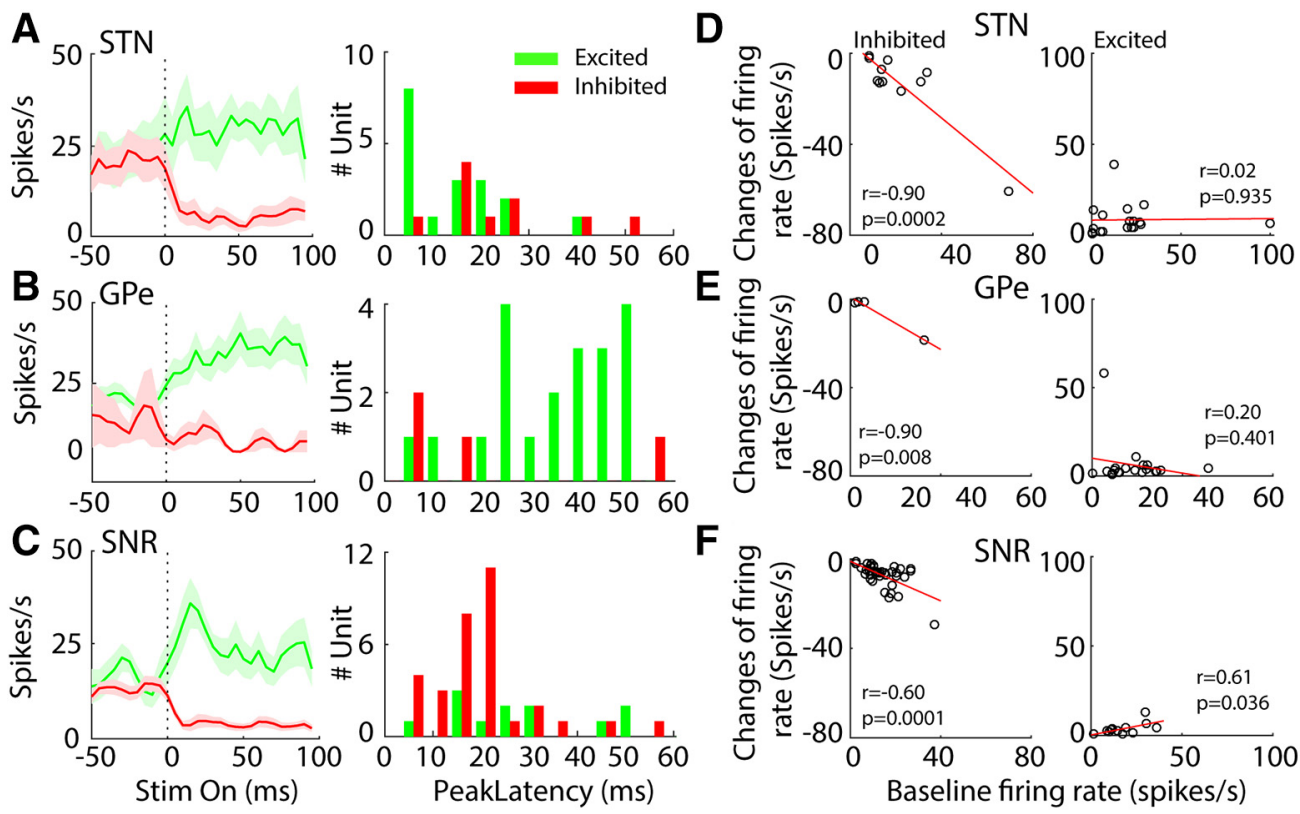

Figure 9. Characteristics of STN, GPe, and SNr neural responses during 130-pps optogenetic STN DBS. A, left, Overall average PSTHs (5-ms bin width) of excited and inhibited STN neurons. Right, Peak response latencies of excited STN neurons and trough latencies of inhibited STN neurons measured from the PSTHs within $100 \mathrm{~ms}$ after stimulus onset. $\boldsymbol{B}$, left, Overall average PSTHs of the excited and inhibited GPe neurons. Right, Peak response latencies of GPe neurons measured from the PSTHs within $100 \mathrm{~ms}$ after stimulus onset. C, left, Overall average PSTHs of excited and inhibited SNr neurons. Right, Peak/trough response latencies of SNr neurons. D. Correlations between changes in firing rate and baseline firing rate in inhibited (left, $n=11$ ) and excited (right, $n=18$ ) STN neurons. $\boldsymbol{E}$, Correlation between change in firing rate and baseline firing rate in inhibited $(n=4)$ and excited $(n=20)$ GPe neurons. $\boldsymbol{F}$, Correlation between change in firing rate and baseline firing rate in inhibited ( $n=37)$ and excited $(n=12)$ SNr neurons.

optical STN DBS (low $\beta, t_{(51)}=5.25, p<0.001$; high $\beta, t_{(51)}=$ $6.07, p<0.001$ ), while no significant reductions were found in GPe for either $\beta$ band (low $\beta, t_{(24)}=0.29, p=0.775$; high $\beta$, $\left.t_{(24)}=0.13, p=0.896\right)$.

To quantify the relationship between changes in neural firing rate and changes in oscillatory activity during STN DBS, we conducted two-way ANOVA of neuronal response type based on changes in firing rates (excited, inhibited, and non-responsive) and changes in oscillatory power (prestimulation vs stimulation). There were no significant interactions between firing rate response and changes in oscillatory power in the $\operatorname{STN}\left(F_{(2,57)}=\right.$ $197.8, p=0.332), \operatorname{SNr}\left(F_{(2,121)}=106.8, p=0.102\right)$, or $\mathrm{GPe}$ $\left(F_{(2,75)}=45.1, p=0.542\right)$ during optogenetic STN DBS.

\section{Effects of optogenetic STN DBS rate on STN and SNr neuronal activity}

We recorded STN $(n=36)$ and $\mathrm{SNr}(n=59)$ neurons during different rates of optogenetic STN DBS. 12/36 STN neurons were recorded during all five rates $(5,20,75,100$, and $130 \mathrm{pps})$, while 24 STN neurons were recorded during DBS at four rates (20, 75,100 , and 130 pps). In SNr, 43 neurons were recorded during DBS with all five rates and 16 neurons were recorded during DBS with four rates.

\section{Neural spiking}

The changes in firing rates in STN and SNr, both increases and decreases in rate, were accentuated at higher rates of optogenetic STN DBS (Fig. 11). However, population data analysis showed that there were no differences in the mean firing rates between baseline and stimulation in STN across stimulation rates (twoway ANOVA with stimulus rate and stimulation on-off as factors, rate $F_{(4,253)}=0.23, p=0.920$, stimulation $F_{(1,253)}=1.06$, $p=0.304$ and no significant interaction between these factors $\left.F_{(4,253)}=0.12, p=0.977\right)$. While there was a significant reduction in mean firing rate of $\mathrm{SNr}$ neurons during stimulation (two-way ANOVA, significant effect with stimulation $F_{(1,419)}=6.06$, $p=0.014)$, there was no significant effect of stimulation rate $\left(F_{(4,419)}=0.07, p=0.992\right)$, and no significant interaction $\left(F_{(4,419)}\right.$ $=0.22, p=0.927)$. However, since DBS produced both increases and decreases in firing rate in different neurons (Figs. 7, 8), there were significant effects of stimulation rate on the absolute changes of firing rate in responsive neurons in both STN (Fig. $11 C ; n=29$, one-way ANOVA with stimulation rate as factor, $F_{(4,126)}=2.97, p=0.02$ ) and SNr (Fig. $11 D ; n=45$, one-way ANOVA, $\left.F_{(4,209)}=9.52, p<0.001\right)$.

\section{Oscillatory activity}

We computed the spectral power of single unit firing to quantify the effects of optogenetic STN DBS rate on oscillatory activity (Fig. 12A,B). Before stimulation, the power spectra of 19 STN neurons (19/36, 53\%) and 37 SNR neurons (37/59, 63\%) showed a pronounced peak in the $\beta$ frequency band $(12-30 \mathrm{~Hz})$. Most of neurons in STN [5 pps, 8/12 (67\%); 20 pps, 14/19 (74\%); 75 pps, $15 / 19$ (79\%); 100 pps, $15 / 19$ (79\%); 130 pps14/19 (74\%) and SNr (5 pps, $16 / 25$ (64\%); 20 pps, $25 / 37$ (68\%); 75 pps, $25 / 37$ (68\%); 100 pps, $25 / 37$ (68\%); 130 pps $24 / 37$ (65\%)] exhibited a significant reduction of $\beta$ band power during stimulation at different rates of optogenetic DBS (paired $t$ test, all $p<0.05$ ). Further, higher-rate optical stimulation produced stronger reductions in neural $\beta$ band oscillatory activity in both STN (one-way ANOVA, $\left.F_{(4,90)}=4.99, p=0.001\right)$ and $\operatorname{SNr}\left(F_{(4,180)}=\right.$ $8.26, p<0.001)$ in comparison to lower rates, as indicated by the ratio of $\beta$ band power during stimulation to prestimulation (Fig. $12 C, D)$.

\section{Discussion}

We quantified effects on motor behavior and neuronal activity of optogenetic STN DBS in the unilateral 6-OHDA lesioned rat 


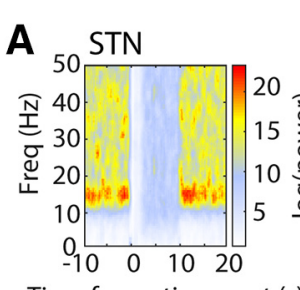

Time from stim onset $(s)$

D
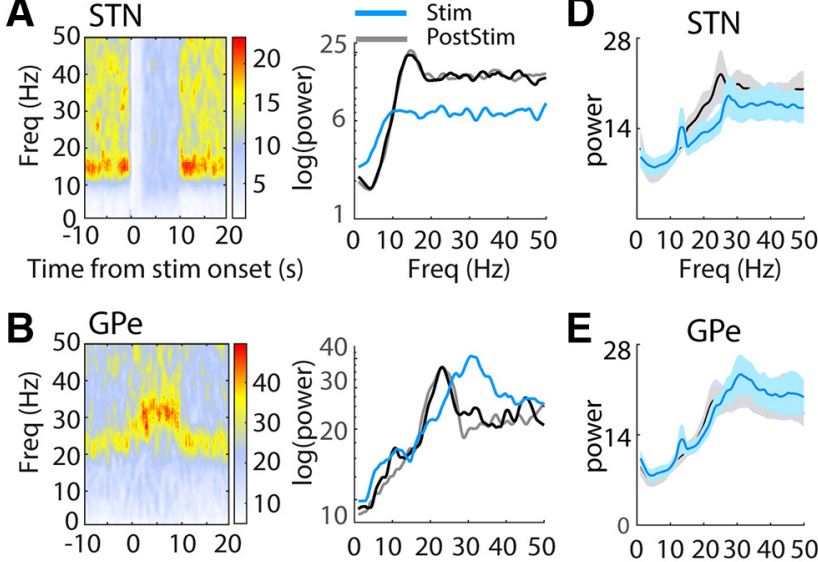

Time from stim onset (s)
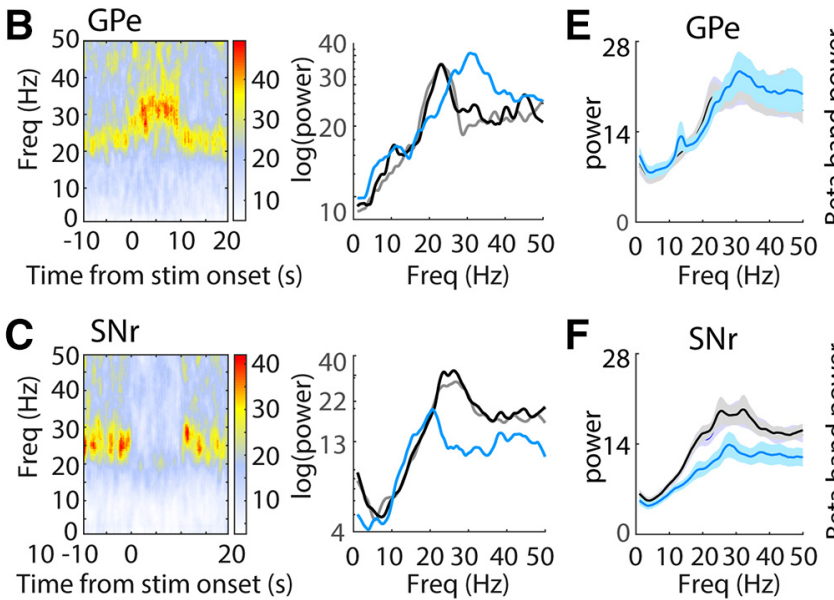

Figure 10. Changes of neural oscillatory activity in the STN, GPe, and SNr during 130-pps optogenetic STN DBS. $A$, left, Representative time-frequency spectrogram shows effect of optogenetic STN DBS on oscillatory activity in a representative STN neuron. Right, Power spectra of the representative single unit spike times during prestimulation (PreStim), stimulation (Stim), and poststimulation (PostStim) periods. $\boldsymbol{B}$, left, Representative time-frequency spectrogram shows effect of optogenetic STN DBS on oscillatory activity in a representative GPe neuron. Right, Power spectra of the representative single unit spike times during prestimulation, stimulation, and poststimulation periods. C, left, Time-frequency spectrogram shows effect of optogenetic STN DBS on oscillatory activity in a representative SNr neuron. Right, Power spectra of the representative $\mathrm{SNr}$ single unit spike times during prestimulation, stimulation, and poststimulation periods. $\boldsymbol{D}-\boldsymbol{F}$, Population power spectra of STN $(\boldsymbol{D}), \mathrm{GPe}(\boldsymbol{E})$, and SNr $(\boldsymbol{F})$ neurons. Optogenetic STN DBS at $130 \mathrm{pps}$ reduced the $\beta$ band oscillatory activity in STN $\left(t_{(25)}=2.10, p=0.044\right.$, paired $t$ test $)$ and $S N r\left(t_{(50)}=6.59, p<0.001\right)$, but not in GPe $\left(t_{(24)}=-0.35, p=0.728\right)$. ${ }^{*} p<0.05$. Data are mean $\pm \mathrm{SE}$.

using an ultrafast opsin, Chronos, that can follow the high rates required for effective DBS (Hight et al., 2015). A CaMKII promoter generated robust expression of Chronos-GFP in glutamatergic STN neurons and their projections in GPe and SNr. Highrate optogenetic STN DBS, with the kinetically-appropriate Chronos construct, alleviated ipsilateral circling bias and increased use of the impaired forelimb. As with electrical DBS, behavioral effects of optogenetic STN DBS were strongly dependent on stimulation rate. Further, we confirmed prior results that high-rate optogenetic STN DBS with kinetically-slower ChR2 was not effective in treating parkinsonism.

Optogenetic STN DBS resulted in both increases and decreases in firing rates of neurons within STN, SNr and GPe, and the absolute changes in rates were accentuated at higher rates of stimulation. $\beta$ Band oscillatory activity was observed in most neurons in STN, GPe, and $\mathrm{SNr}$, and high-rate optogenetic STN DBS suppressed $\beta$ band oscillatory activity within the STN and SNr. This suppression was DBS rate dependent, and high rates produced stronger suppression. Changes in $\beta$ power were not correlated with changes in firing rates, implying that the efficacy of DBS is due to suppression of pathologic oscillatory activity, rather than generation of specific changes in firing rate.

Multiple neural elements may be affected by DBS and mediate therapeutic effects, while others may mediate side effects. Determining which circuits contribute to effective DBS requires
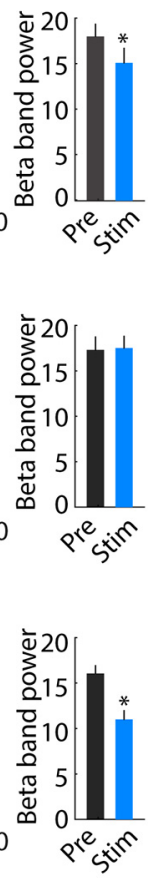

manipulating selectively the activity of specific neural elements. Optogenetics provides cell-type specificity and was used previously to determine the neural elements contributing to symptom relief by STN DBS in parkinsonian rodents (Gradinaru et al., 2009). That study concluded that activation of STN local cells was neither necessary nor sufficient to relieve parkinsonian motor dysfunction. However, the effects of DBS are strongly dependent on the stimulation rate, and the slow kinetics of ChR2 precluded following high-rate stimulation (>100 pps) necessary for effectiveness, as high-rate laser pulses did not result in faithful high-rate neural activity (Klapoetke et al., 2014; Hight et al., 2015; Jun and Cardin, 2020). Therefore, it was critical to determine the role of STN local cells in the symptom-relieving effects of STN DBS using faster opsins. Optogenetic activation of Chronos-expressing STN neurons at $130 \mathrm{pps}$ reduced pathologic circling and improved forelimb stepping, consistent with effects of electrical DBS. Chronos exhibits higher overall driven firing rates and greater firing rate fidelity than ChR2, as well as greater sensitivity to illumination (Yizhar et al., 2011; Klapoetke et al., 2014; Hight et al., 2015; Jun and Cardin, 2020). Thus, it is unlikely that the behavioral differences between ChR2 and Chronos resulted from differences in the intensity of light required to elicit neuron activation, but rather that they resulted from the differences in the kinetic properties of the opsins. These results highlight the importance of considering the kinetic limits of opsins when probing the effects and mechanisms of DBS (Gradinaru et al., 2009; Lin et al., 2011; Sanders and Jaeger, 2016; Servello et al., 2016; Ordaz et al., 2017).

The relief of pathologic circling during electrical DBS was greater than during DBS using Chronos, which may suggest that STN local cell-specific activation may not produce complete alleviation of motor symptoms. Antidromic cortical input may be engaged by electrical DBS and enhance therapeutic efficacy, consistent with the hypothesis of DBS antidromic activation in M1 (Gradinaru et al., 2009; Li et al., 2012; Sanders and Jaeger, 2016). In addition, optogenetic responses depend on opsin expression, and increasing the opsin expression level could enhance optogenetic excitability (Meng et al., 2019) and improve behavioral outcomes. Thus, the difference between optogenetic and electrical effects on circling may be due to insufficient opsin expression and/or limited light illumination area (Yizhar et al., 2011). Differences in baseline circling across animals may reflect differences in the extent of dopaminergic lesion and/or differences in sensitivity to methamphetamine (So et al., 2012).

Changes in neural firing rates and patterns in the basal ganglia are associated with parkinsonism and are altered by DBS (Raz et al., 2000; Goldberg et al., 2002, 2004; Bar-Gad et al., 2004; Hammond et al., 2007; Erez et al., 2009; Chiken and Nambu, 2013; Little and Brown, 2014). Previous studies reported different effects of electrical DBS on neural firing rate. Some studies 
showed that STN DBS resulted in an increase in GPe and GPi rates (Hashimoto et al., 2003), while others reported a reduction of GPi and SNr activity (Benazzouz et al., 1995, 2000). Consistent with our results with optogenetic DBS, several studies demonstrated that STN DBS produced both increases and decreases in rates in the GPe, $\mathrm{SNr}$, and striatum, and that there were no significant changes in the population mean firing rates (Shi et al., 2006; Bosch et al., 2011; McConnell et al., 2012). Like electrical stimulation, optogenetic stimulation can generate complex responses at the stimulation site, despite selective activation of specific cell types (Han et al., 2009; Galvan et al., 2012), and optogenetic STN DBS both increased and decreased firing rates of STN neurons consistent with previous findings in optogenetic studies of striatal and cortical neurons (Han et al., 2009; Galvan et al., 2012). The inhibition of STN neurons occurred immediately after light stimulation without prior activation. Further, latencies of inhibited neurons were longer than those of excited neurons, and the reduced firing rates of inhibited neurons were significantly correlated with their baseline firing rates. These observations support that the reduction of firing rates in neurons during light stimulation resulted from synaptic activation of downstream inhibitory neurons that suppressed the recorded excitatory neurons.

Optogenetic STN stimulation generated both activation and inhibition in $\mathrm{SNr}$ and GPe neurons, with most $\mathrm{SNr}$ neurons being inhibited and most GPe neurons being activated. The latencies of both inhibited and excited $\mathrm{SNr}$ neurons were longer than those of STN excited neurons, consistent with effects in $\mathrm{SNr}$ being mediated transsynaptically. While excitation of SNr neurons was most likely caused by glutamatergic input from the STN, suppression of $\mathrm{SNr}$ neurons could result from network mechanisms involving both local and external inhibitory inputs. Similar to inhibited neurons in STN, inhibition of SNr neurons occurred promptly on light stimulation without prior activation, and reductions in firing rate were dependent on baseline firing rates, suggesting the involvement of local inhibitory networks. Further, activation of more neurons in GPe may generate additional GABAergic inhibitory input to $\mathrm{SNr}$ (Windels et al., 2005), and this hypothesis supports a role of GPe-SNr connections in mediating the effects of STN DBS (Windels et al., 2000, 2003).

Changes in neural firing patterns play an important role in the pathophysiology of PD (Hamani and Lozano, 2003; Wichmann and DeLong, 2003). Dopamine depletion induces abnormal neural activity in the basal ganglia, and $\beta$ oscillatory activity is implicated in the pathophysiology of PD (Hammond et al., 2007). $\beta$ Band activity can be subdivided into low (13$20 \mathrm{~Hz}$ ) and high $(20-30 \mathrm{~Hz})$ frequencies (Priori et al., 2004), with low $\beta$ band activity more strongly correlated with rigidity and
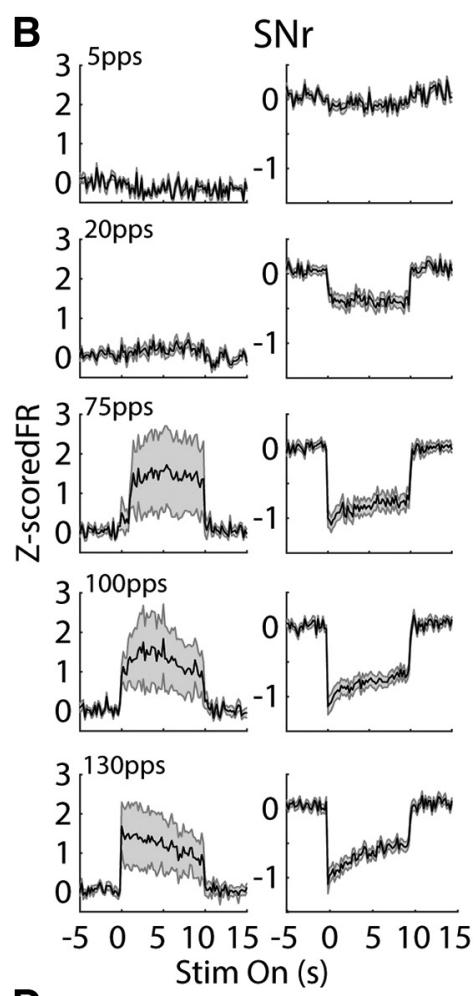

D

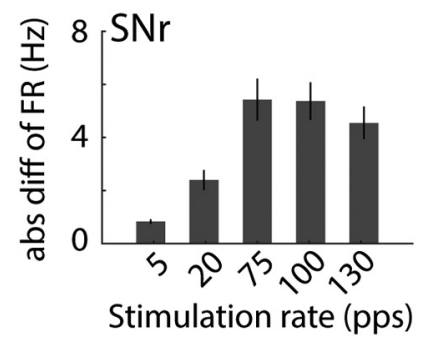

Figure 11. Effects of optogenetic STN DBS rate on neural population firing rates in STN and SNr. $\boldsymbol{A}$, Mean poststimulus histograms of STN neurons that were excited (left) or inhibited (right) by 130-pps stimulation in response to optogenetic 100 , and $130 \mathrm{pps}$. $\boldsymbol{C}, \boldsymbol{D}$, Absolute changes of firing rate in responsive neurons in $\operatorname{STN}(\boldsymbol{C}, n=29)$ and $\operatorname{SNr}(\boldsymbol{D}, n=45)$ at different stimulation rates. One-way ANOVA, STN: $F_{(4,126)}=2.97, p=0.02 ;$ SNr: $\left.F_{(4,209)}=9.52, p<0.001\right)$.

bradykinesia, and high $\beta$ power elevated in patients with freezing of gait (Toledo et al., 2014). Further, $\beta$ activity appears to shift to lower frequencies with increased dopamine depletion (Connolly et al., 2015), and low $\beta$ activity is more sensitive than high $\beta$ to dopaminergic treatment (Priori et al., 2004; LópezAzcárate et al., 2010). Collectively, these results suggest that differential changes in the low and high $\beta$ bands may have differential effects on parkinsonian symptoms.

Previous studies with electrical DBS indicated that therapeutic effects result from overriding pathologic neural activity and disrupting abnormal neural firing patterns (Hashimoto et al., 2003; Grill et al., 2004; Dorval et al., 2008, 2010; Rosin et al., 2011; McCairn and Turner, 2015; McConnell et al., 2016; So et al., 2017). Consistent with these findings, optogenetic STN DBS suppressed $\beta$ band activity in STN and SNr, and effects were dependent on the stimulation rate. High-rate optical DBS strongly reduced $\beta$ oscillatory activity, while low rates had smaller effects or were ineffective. We quantified the effects of STN optogenetic DBS on both low and high $\beta$ oscillatory activity. High $\beta$ activity of STN neurons was reduced during optogenetic STN DBS, while 


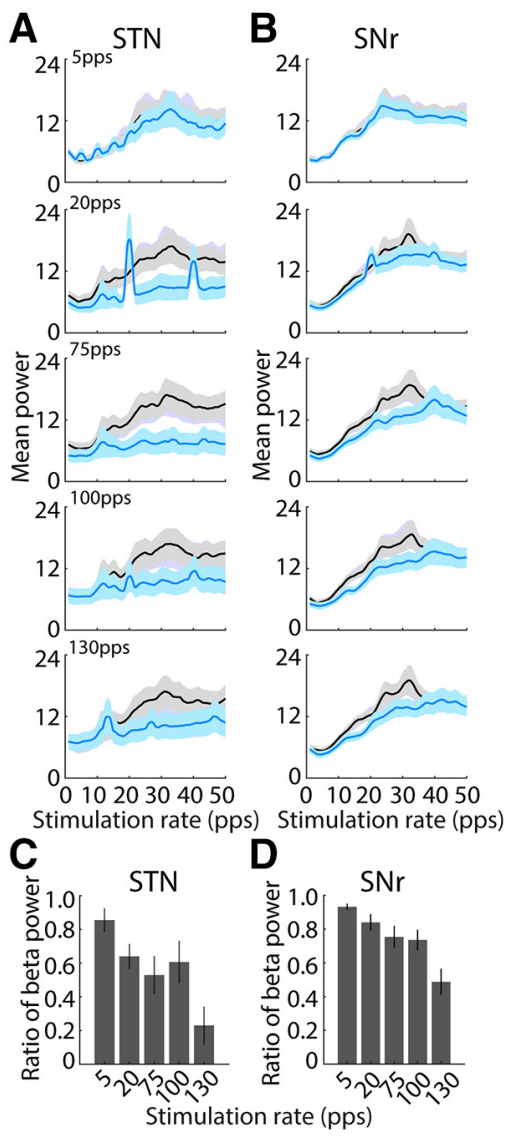

Figure 12. Effects of optogenetic STN DBS rate on neural oscillatory activity in STN and SNr during STN. A, Population power spectra of STN oscillatory unit spike times during prestimulation (black) and stimulation (blue), periods at each stimulation rate. Optogenetic stimulation at higher rates $(75,100$, and $130 \mathrm{pps})$ remarkably suppressed $\beta$ band oscillations in STN, but lower rates did not. $\boldsymbol{B}$, Population power spectra of SNr oscillatory unit spike times during prestimulation and stimulation, periods at each rate. STN optical stimulation at higher rates $(75,100$, and $130 \mathrm{pps})$ predominately reduced $\beta$ band oscillations in $\mathrm{SNr}$, while lower rates did not. $\mathbf{C}$, Normalized $\beta$ band $(13-30 \mathrm{~Hz})$ power of STN oscillatory neurons $(n=19)$ at different stimulation rates. The reduction of $\beta$ oscillatory activities was increased at higher rates (one-way ANOVA, $\left.F_{(4,90)}=4.99, p=0.001\right)$. D. Normalized $\beta$ band $(13-30 \mathrm{~Hz}$ ) power of SNr oscillatory neurons $(n=37)$ at different rates. The reduction of $\beta$ oscillatory activities was also increased at higher rates (one-way ANOVA, $F_{(4,180)}=8.26 p<0.001$ ).

low $\beta$ activity was unchanged. Meanwhile, both low and high $\beta$ activity of $\mathrm{SNr}$ neurons was dramatically reduced, while there were no changes in the $\beta$ oscillatory activity in GPe. Interestingly, the oscillatory activity of some GPe and STN neurons shifted from low $\beta$ to high $\beta$ during STN optogenetic DBS, and this may contribute to symptom relief. Further, the changes in oscillatory activity in STN and SNr neurons were independent of changes in firing rate, supporting the hypothesis that the therapeutic effect of DBS resulted from suppressing pathologic oscillatory activity. Cell-specific optogenetic activation of STN local neurons was thus sufficient to alter neural firing patterns within basal ganglia circuits and ameliorate motor symptoms in parkinsonian rats.

\section{References}

Albaugh DL, Shih YY (2014) Neural circuit modulation during deep brain stimulation at the subthalamic nucleus for Parkinson's disease: what have we learned from neuroimaging studies? Brain Connect 4:1-14.
Alexander GE, Crutcher MD, DeLong MR (1990) Basal ganglia-thalamocortical circuits: parallel substrates for motor, oculomotor, "prefrontal" and "limbic" functions. Prog Brain Res 85:119-146.

Bar-Gad I, Elias S, Vaadia E, Bergman H (2004) Complex locking rather than complete cessation of neuronal activity in the globus pallidus of a 1methyl-4-phenyl-1,2,3,6-tetrahydropyridine-treated primate in response to pallidal microstimulation. J Neurosci 24:7410-7419.

Benazzouz A, Piallat B, Pollak P, Benabid AL (1995) Responses of substantia nigra pars reticulata and globus pallidus complex to high frequency stimulation of the subthalamic nucleus in rats: electrophysiological data. Neurosci Lett 189:77-80.

Benazzouz A, Gao DM, Ni ZG, Piallat B, Bouali-Benazzouz R, Benabid AL (2000) Effect of high-frequency stimulation of the subthalamic nucleus on the neuronal activities of the substantia nigra pars reticulata and ventrolateral nucleus of the thalamus in the rat. Neuroscience 99:289-295.

Bosch C, Degos B, Deniau JM, Venance L (2011) Subthalamic nucleus highfrequency stimulation generates a concomitant synaptic excitation-inhibition in substantia nigra pars reticulata. J Physiol 589:4189-4207.

Boyden ES, Zhang F, Bamberg E, Nagel G, Deisseroth K (2005) Millisecondtimescale, genetically targeted optical control of neural activity. Nat Neurosci 8:1263-1268.

Chiken S, Nambu A (2013) High-frequency pallidal stimulation disrupts information flow through the pallidum by GABAergic inhibition. J Neurosci 33:2268-2280.

Connolly AT, Jensen AL, Bello EM, Netoff TI, Baker KB, Johnson MD, Vitek JL (2015) Modulations in oscillatory frequency and coupling in globus pallidus with increasing parkinsonian severity. J Neurosci 35:6231-6240.

Deep-Brain Stimulation for Parkinson's Disease Study Group, Obeso JA, Olanow CW, Rodriguez-Oroz MC, Krack P, Kumar R, Lang AE (2001) Deep-brain stimulation of the subthalamic nucleus or the pars interna of the globus pallidus in Parkinson's disease. N Engl J Med 345:956-963.

Dorval AD, Russo GS, Hashimoto T, Xu W, Grill WM, Vitek JL (2008) Deep brain stimulation reduces neuronal entropy in the MPTP-primate model of Parkinson's disease. J Neurophysiol 100:2807-2818.

Dorval AD, Kuncel AM, Birdno MJ, Turner DA, Grill WM (2010) Deep brain stimulation alleviates parkinsonian bradykinesia by regularizing pallidal activity. J Neurophysiol 104:911-921.

Erez Y, Czitron H, McCairn K, Belelovsky K, Bar-Gad I (2009) Short-term depression of synaptic transmission during stimulation in the globus pallidus of 1-methyl-4-phenyl-1,2,3,6-tetrahydropyridine-treated primates. J Neurosci 29:7797-7802.

Galvan A, Hu X, Smith Y, Wichmann T (2012) In vivo optogenetic control of striatal and thalamic neurons in non-human primates. PLoS One 7: e50808.

Ghasemi A, Zahediasl S (2012) Normality tests for statistical analysis: a guide for non-statisticians. Int J Endocrinol Metab 10:486-489.

Glajch KE, Fleming SM, Surmeier DJ, Osten P (2012) Sensorimotor assessment of the unilateral 6-hydroxydopamine mouse model of Parkinson's disease. Behav Brain Res 230:309-316.

Goldberg JA, Boraud T, Maraton S, Haber SN, Vaadia E, Bergman H (2002) Enhanced synchrony among primary motor cortex neurons in the 1-methyl-4-phenyl-1,2,3,6-tetrahydropyridine primate model of Parkinson's disease. J Neurosci 22:4639-4653.

Goldberg JA, Rokni U, Boraud T, Vaadia E, Bergman H (2004) Spike synchronization in the cortex/basal-ganglia networks of Parkinsonian primates reflects global dynamics of the local field potentials. J Neurosci 24:6003-6010.

Gradinaru V, Mogri M, Thompson KR, Henderson JM, Deisseroth K (2009) Optical deconstruction of parkinsonian neural circuitry. Science 324:354-359.

Grill WM, Snyder AN, Miocinovic S (2004) Deep brain stimulation creates an informational lesion of the stimulated nucleus. Neuroreport 15:11371140.

Hamani C, Lozano AM (2003) Physiology and pathophysiology of Parkinson's disease. Ann NY Acad Sci 991:15-21.

Hammond C, Bergman H, Brown P (2007) Pathological synchronization in Parkinson's disease: networks, models and treatments. Trends Neurosci 30:357-364.

Han X, Qian X, Bernstein JG, Zhou HH, Franzesi GT, Stern P, Bronson RT, Graybiel AM, Desimone R, Boyden ES (2009) Millisecond-timescale optical control of neural dynamics in the nonhuman primate brain. Neuron 62:191-198. 
Hashimoto T, Elder CM, Okun MS, Patrick SK, Vitek JL (2003) Stimulation of the subthalamic nucleus changes the firing pattern of pallidal neurons. J Neurosci 23:1916-1923.

Hight AE, Kozin ED, Darrow K, Lehmann A, Boyden E, Brown MC, Lee DJ (2015) Superior temporal resolution of Chronos versus channelrhodopsin-2 in an optogenetic model of the auditory brainstem implant. Hearing Res 322:235-241.

Jun NY, Cardin JA (2020) Activation of distinct Channelrhodopsin variants engages different patterns of network activity. eNeuro 7:ENEURO.022218.2019.

Klapoetke NC, Murata Y, Kim SS, Pulver SR, Birdsey-Benson A, Cho YK, Morimoto TK, Chuong AS, Carpenter EJ, Tian Z, Wang J, Xie Y, Yan Z, Zhang Y, Chow BY, Surek B, Melkonian M, Jayaraman V, ConstantinePaton M, Wong GK, et al. (2014) Independent optical excitation of distinct neural populations. Nat Methods 11:338-346.

Krack P, Pollak P, Limousin P, Hoffmann D, Xie J, Benazzouz A, Benabid AL (1998) Subthalamic nucleus or internal pallidal stimulation in young onset Parkinson's disease. Brain 121:451-457.

Li Q, Ke Y, Chan DC, Qian ZM, Yung KK, Ko H, Arbuthnott GW, Yung WH (2012) Therapeutic deep brain stimulation in Parkinsonian rats directly influences motor cortex. Neuron 76:1030-1041.

Lin JY, Lin MZ, Steinbach P, Tsien RY (2009) Characterization of engineered channelrhodopsin variants with improved properties and kinetics. Biophys J 96:1803-1814.

Lin SC, Deisseroth K, Henderson JM (2011) Optogenetics: background and concepts for neurosurgery. Neurosurgery 69:1-3.

Little S, Brown P (2014) The functional role of beta oscillations in Parkinson's disease. Parkinsonism Relat Disord 20 [Suppl 1]:S44-S48.

López-Azcárate J, Tainta M, Rodríguez-Oroz MC, Valencia M, González R, Guridi J, Iriarte J, Obeso JA, Artieda J, Alegre M (2010) Coupling between beta and high-frequency activity in the human subthalamic nucleus may be a pathophysiological mechanism in Parkinson's disease. J Neurosci 30:6667-6677.

McCairn KW, Turner RS (2015) Pallidal stimulation suppresses pathological dysrhythmia in the parkinsonian motor cortex. J Neurophysiol 113:2537-2548.

McConnell GC, So RQ, Hilliard JD, Lopomo P, Grill WM (2012) Effective deep brain stimulation suppresses low-frequency network oscillations in the basal ganglia by regularizing neural firing patterns. J Neurosci 32:15657-15668.

McConnell GC, So RQ, Grill WM (2016) Failure to suppress low-frequency neuronal oscillatory activity underlies the reduced effectiveness of random patterns of deep brain stimulation. J Neurophysiol 115:2791-2802.

Meng X, Murali S, Cheng YF, Lu J, Hight AE, Kanumuri VV, Brown MC, Holt JR, Lee DJ, Edge ASB (2019) Increasing the expression level of ChR2 enhances the optogenetic excitability of cochlear neurons. J Neurophysiol 122:1962-1974.

Mitra PP, Pesaran B (1999) Analysis of dynamic brain imaging data. Biophys J 76:691-708.

Ordaz JD, Wu W, Xu XM (2017) Optogenetics and its application in neural degeneration and regeneration. Neural Regen Res 12:1197-1209.

Paxinos G, Watson W (2005) The rat brain in stereotaxic coordinates. San Diego: Academic Press.

Priori A, Foffani G, Pesenti A, Tamma F, Bianchi AM, Pellegrini M, Locatelli M, Moxon KA, Villani RM (2004) Rhythm-specific pharmacological modulation of subthalamic activity in Parkinson's disease. Exp Neurol 189:369-379.

Raz A, Vaadia E, Bergman H (2000) Firing patterns and correlations of spontaneous discharge of pallidal neurons in the normal and the tremulous 1methyl-4-phenyl-1,2,3,6-tetrahydropyridine vervet model of parkinsonism. J Neurosci 20:8559-8571.

Rosin B, Slovik M, Mitelman R, Rivlin-Etzion M, Haber SN, Israel Z, Vaadia E, Bergman H (2011) Closed-loop deep brain stimulation is superior in ameliorating parkinsonism. Neuron 72:370-384.

Sanders TH, Jaeger D (2016) Optogenetic stimulation of cortico-subthalamic projections is sufficient to ameliorate bradykinesia in 6-ohda lesioned mice. Neurobiol Dis 95:225-237.

Schallert T, Whishaw IQ, Ramirez VD, Teitelbaum P (1978) Compulsive, abnormal walking caused by anticholinergics in akinetic, 6-hydroxydopamine-treated rats. Science 199:1461-1463.

Servello D, Zekaj E, Saleh C, Lange N, Porta M (2016) Deep brain stimulation in Gilles de la Tourette syndrome: what does the future hold? A cohort of 48 patients. Neurosurgery 78:91-100.

Shi LH, Luo F, Woodward DJ, Chang JY (2006) Basal ganglia neural responses during behaviorally effective deep brain stimulation of the subthalamic nucleus in rats performing a treadmill locomotion test. Synapse 59:445-457.

So RQ, McConnell GC, August AT, Grill WM (2012) Characterizing effects of subthalamic nucleus deep brain stimulation on methamphetamineinduced circling behavior in hemi-Parkinsonian rats. IEEE Trans Neural Syst Rehabil Eng 20:626-635.

So RQ, McConnell GC, Grill WM (2017) Frequency-dependent, transient effects of subthalamic nucleus deep brain stimulation on methamphetamine-induced circling and neuronal activity in the hemiparkinsonian rat. Behav Brain Res 320:119-127.

Sparta DR, Stamatakis AM, Phillips JL, Hovelsø N, van Zessen R, Stuber GD (2011) Construction of implantable optical fibers for long-term optogenetic manipulation of neural circuits. Nat Protoc 7:12-23.

Toledo JB, López-Azcárate J, Garcia-Garcia D, Guridi J, Valencia M, Artieda J, Obeso J, Alegre M, Rodriguez-Oroz M (2014) High beta activity in the subthalamic nucleus and freezing of gait in Parkinson's disease. Neurobiol Dis 64:60-65.

Wichmann T, DeLong MR (2003) Pathophysiology of Parkinson's disease: the MPTP primate model of the human disorder. Ann NY Acad Sci 991:199-213.

Windels F, Bruet N, Poupard A, Urbain N, Chouvet G, Feuerstein C, Savasta M (2000) Effects of high frequency stimulation of subthalamic nucleus on extracellular glutamate and GABA in substantia nigra and globus pallidus in the normal rat. Eur J Neurosci 12:4141-4146.

Windels F, Bruet N, Poupard A, Feuerstein C, Bertrand A, Savasta M (2003) Influence of the frequency parameter on extracellular glutamate and gamma-aminobutyric acid in substantia nigra and globus pallidus during electrical stimulation of subthalamic nucleus in rats. J Neurosci Res 72:259-267.

Windels F, Carcenac C, Poupard A, Savasta M (2005) Pallidal origin of GABA release within the substantia nigra pars reticulata during high-frequency stimulation of the subthalamic nucleus. J Neurosci 25:5079-5086.

Yizhar O, Fenno LE, Davidson TJ, Mogri M, Deisseroth K (2011) Optogenetics in neural systems. Neuron 71:9-34 